\title{
Staging systems and nomograms in soft tissue sarcoma: outcome prediction by categorization or personalization?
}

\author{
William W. Tseng ${ }^{1}$, Sandro Pasquali ${ }^{2}$, James S. Hu ${ }^{3}$, Lawrence R. Menendez ${ }^{4}$, Alessandro Gronchi ${ }^{2}$ \\ ${ }^{1}$ Department of Surgery, University of Southern California, Keck School of Medicine, Los Angeles, CA, USA; ${ }^{2}$ Department of Surgery, Fondazione \\ IRCCS, Istituto Nazionale dei Tumori, Milan, Italy; ${ }^{3}$ Department of Medical Oncology, ${ }^{4}$ Department of Orthopedic Surgery, University of Southern \\ California, Keck School of Medicine, Los Angeles, CA, USA \\ Correspondence to: William W. Tseng, MD. Associate Professor of Surgery, USC Surgical Oncology, 1510 San Pablo Street, HCC-I, Suite 514, Los \\ Angeles, CA USA. Email: william.tseng@med.usc.edu. \\ Comment on: Johnson AC, Ethun CG, Liu Y, et al. A novel, simplified, externally validated staging system for truncal/extremity soft tissue sarcomas: \\ An analysis of the US Sarcoma Collaborative database. J Surg Oncol 2018;118:1135-41.
}

Submitted Jan 12, 2019. Accepted for publication Jan 18, 2019.

doi: $10.21037 /$ cco. 2019.01 .05

View this article at: http://dx.doi.org/10.21037/cco.2019.01.05

Soft tissue sarcomas (STS) are rare cancers that most commonly develop in the extremities and trunk, but can develop in any location throughout the body. Similar to other cancers, staging systems have been available, but for STS these have historically been criticized as inadequate and poorly applicable to daily clinical practice. In 2018, the American Joint Cancer Committee's (AJCC) TNM staging system was updated $\left(8^{\text {th }}\right.$ edition) with changes such as separation by tumor location and categorization of all nodepositive disease as Stage 4.

In a recent study by Johnson et al., a more simplified staging system for extremity and truncal STS was proposed (1). When the authors applied the AJCC $8^{\text {th }}$ edition to their own combined cohort of over 1,300 STS patients from seven academic institutions in the United States (US), they found poor discrimination of clinical outcome for those categorized as Stage $1 \mathrm{~A}$ versus $1 \mathrm{~B}$ and Stage 2 versus $3 \mathrm{~A}$. The authors then re-analyzed the data from their cohort and found better outcome discrimination when all patients with low grade disease were categorized as Stage 1 and those with high grade disease were stratified to Stage 2 and 3 based on a tumor size cutoff of $<$ or $>7.5 \mathrm{~cm}$. Patients with distant metastatic disease remained Stage 4. This novel staging system was then externally validated using the US National Cancer Database (NCDB), which encompasses over 9,000 patients, and was found to be non-inferior in prognostic stratification compared to the AJCC $8^{\text {th }}$ edition $(\mathrm{C}$-index $=0.727)$.
While the staging system from Johnson et al. is indeed simpler, STS is inherently complex given not only the variety of body locations but also importantly, the heterogeneity of histologic subtypes that exist. Currently over 50-70 distinct subtypes are recognized-we have previously even made the analogy to varieties of wine (2). To develop their staging system, Johnson et al. excluded several histologic subtypes of STS. Some of these may be justified (e.g., desmoid tumors, which do not metastasize and may even spontaneously regress); however, the rationale for excluding other subtypes is less clear (e.g., angiosarcoma, malignant peripheral nerve sheath tumor-both commonly seen in the extremities and trunk). Ultimately, the proposed staging system, similar to the AJCC $8^{\text {th }}$ edition, excludes histologic subtype all together. For validation, the most common subtypes included in the developmental cohort do not match with those in the validation cohort (NCDB). In fact, strangely, the third most common subtype for NCDB is "giant cell sarcoma" which is actually a malignancy of bone, not soft tissue.

Histologic subtype is very much clinically relevant, even as an independent variable, for the management of STS in daily clinical practice. In fact, especially for nonspecialists who may not appreciate the nuances of STS, clinical-decision making is impaired without recognition of histologic subtype. For example, even within liposarcoma, an STS of fat origin, there are three different subtypes (well differentiated/dedifferentiated, myxoid round cell 


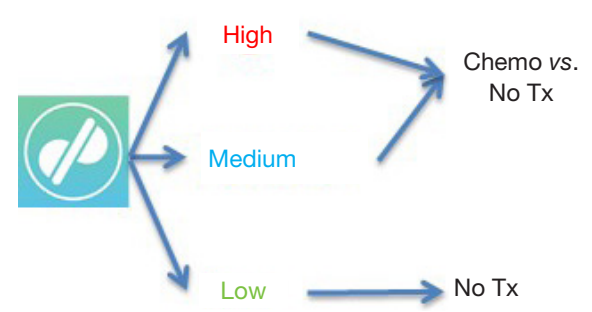

Figure 1 A proposed example of use of the sarculator to prospectively risk stratify patients to different treatment groups for a clinical trial.

and pleomorphic) that each have distinct genetic features, clinical behavior, treatment sensitivities and clinical outcome. To categorize a high grade, $>7.5 \mathrm{~cm}$ extremity STS simply as Stage 3 seems to be incomplete without distinguishing it as undifferentiated pleomorphic sarcoma (UPS) and not for example, myxoid liposarcoma, which could potentially metastasize to other extrapulmonary fat-bearing areas of the body and is uniquely sensitive to radiation and systemic therapy.

Since the early 2000s, partly in response to the criticism against staging systems, nomograms have been reported which are designed to provide personalized predictions of clinical outcome based on a given patient's demographics and tumor characteristics (3). Nomograms exists for STS in general as well as for specific body location and even histologic subtypes (e.g., liposarcoma, synovial sarcoma). An example of an extremity and truncal STS nomogram was reported by Callegaro et al. in 2016 (4). Data from one high volume sarcoma center (over 1,400 patients) was used to develop two nomograms for survival and distant metastasis. Similar to the study by Johnson et al., the authors then externally validated these nomograms, in this case with data from three other sarcoma centers ( 2,300 patients combined). Along with grade and tumor size, histologic subtype was found to be a significant variable and incorporated into the nomograms. Ageanother variable highly relevant to decision-making in clinical practice-was also incorporated into the nomogram for overall survival. These and nomograms for other body locations are now available to clinicians as the "Sarculator," a free application that can be downloaded to any smartphone (https://www.sarculator.com/).

Currently, outcome prediction and risk stratification by either staging systems or nomograms can be used for patient counseling and to help guide treatment planning. In fact, they may even be complementary. Importantly, neither should be used without clinical judgement (e.g., consideration of patient co-morbidities) and ideally, these tools should be used in the context of individual or multidisciplinary expertise with STS. There are also important considerations with the data used for staging systems and nomograms such as the fact that determination of tumor grade may be compromised after pretreatment (e.g., radiation or systemic therapy).

Further work is still needed. As STS is, of course, not limited to Western countries, validation remains to be done with other patient populations. STS nomograms developed using data from US/European patients have been studied in Asian patients and were found to have good accuracy and applicability $(5,6)$. Additional global validation is encouraged; this will also have the secondary benefit of encouraging standardization of treatment and overall foster research collaboration, which is critical in STS. Future investigation should also explore the applicability of staging systems and nomograms for STS in varied practice settings such as community $v s$. academic, sarcoma referral (defined by volume) $v s$. non-referral centers, and even across socioeconomic status.

One key question is whether staging systems, nomograms or both can be meaningfully applied to therapeutic clinical trials. Given the rarity of STS, clinical trials in this disease are often challenging logistically and therefore they demand even more stringent design, which includes patient selection and treatment assignment. The nomogram by Callegaro et al. was recently applied to a series of STS patients included in a clinical trial of neoadjuvant chemotherapy (ISG-GEIS) and proven to be very effective in stratifying their prognosis (7). Remarkably, even though the nomogram was originally designed for use in the adjuvant setting, based on data obtained from nonpretreated tumor specimens (core needle biopsies), it was shown to also perform well even in the neoadjuvant setting. In addition, recent secondary analysis of a negative adjuvant trial (EORTC 62931) showed that re-stratification of patients using the Sarculator could actually identify a subset of patients who in fact did benefit from treatment (8). These studies suggest that indeed nomograms may have utility as a tool to prospectively and personally risk stratify patients to be enrolled in a neoadjuvant or adjuvant clinical trial (Figure 1).

In conclusion, Johnson et al. should be commended for their efforts to optimize methods for outcome prediction; however, limitations still exist and currently, whether it is best to categorize (staging systems) or personalize (nomograms) remains to be determined. 


\section{Acknowledgments}

None.

\section{Footnote}

Conflicts of Interest: The authors have no conflicts of interest to declare.

\section{References}

1. Johnson AC, Ethun CG, Liu Y, et al. A novel, simplified, externally validated staging system for truncal/extremity soft tissue sarcomas: An analysis of the US Sarcoma Collaborative database. J Surg Oncol 2018;118:1135-41.

2. Tseng WW, Pollock RE, Gronchi A. Retroperitoneal sarcomas: Big tumors that involve more than just "Getting it Out". J Surg Oncol 2018;117:5-6.

3. Callegaro D, Miceli R, Mariani L, et al. Soft tissue sarcoma nomograms and their incorporation into practice. Cancer 2017;123:2802-20.

4. Callegaro D, Miceli R, Bonvalot S, et al. Development and external validation of two nomograms to predict overall

Cite this article as: Tseng WW, Pasquali S, Hu JS, Menendez LR, Gronchi A. Staging systems and nomograms in soft tissue sarcoma: outcome prediction by categorization or personalization? Chin Clin Oncol 2019;8(Suppl 1):S12. doi: 10.21037/cco.2019.01.05 survival and occurrence of distant metastases in adults after surgical resection of localised soft-tissue sarcomas of the extremities: a retrospective analysis. Lancet Oncol 2016;17:671-80.

5. Chou YS, Liu CY, Chang YH, et al. Prognostic factors of primary resected retroperitoneal soft tissue sarcoma: Analysis from a single asian tertiary center and external validation of Gronchi's nomogram. J Surg Oncol 2016;113:355-60.

6. Ng DWJ, Tan GHC, Chia CS, et al. Is the Memorial Sloan Kettering Cancer Centre (MSKCC) sarcoma nomogram useful in an Asian population? Asia Pac J Clin Oncol 2017;13:e466-72.

7. Pasquali S, Colombo C, Pizzamiglio S, et al. High-risk soft tissue sarcomas treated with perioperative chemotherapy: Improving prognostic classification in a randomised clinical trial. Eur J Cancer 2018;93:28-36.

8. Pasquali S, Pizzamiglio S, Touati N, et al. The impact of chemotherapy on survival of patients with extremity and trunk wall soft tissue sarcoma: revisiting the results of the EORTC-STBSG 62931 randomized trial. Eur J Cancer 2019. [Epub ahead of print]. 